\title{
Detecting Phases in Parallel Applications on Shared Memory Architectures
}

\author{
Erez Perelman ${ }^{\dagger}$ \\ Marzia Polito \\ Jean-Yves Bouguet ${ }^{\ddagger}$ \\ Brad Calder ${ }^{\dagger}$ \\ Carole Dulong ${ }^{\ddagger}$ \\ John Sampson ${ }^{\dagger}$ \\ ${ }^{\dagger}$ Department of Computer Science and Engineering, University of California, San Diego \\ ${ }^{\ddagger}$ Intel Corporation
}

UCSD Technical Report CS2005-0844

\begin{abstract}
Most programs are repetitive, where similar behavior can be seen at different execution times. Algorithms have been proposed that automatically group similar portions of a program's execution into phases, where samples of execution in the same phase have homogeneous behavior and similar resource requirements.

In this paper, we examine applying these phase analysis algorithms and how to adapt them to parallel applications running on shared memory processors. Our approach relies on a separate representation of each thread's activity. We first focus on showing its ability to identify similar intervals of execution across threads for a single run. We then show that it is effective at identifying similar behavior of a program when the number of threads is varied between runs. This can be used by developers to examine how different phases scale across different number of threads. Finally, we examine using the phase analysis to pick simulation points to guide multi-threaded simulation.
\end{abstract}

\section{Introduction}

The behavior of a program is not random - as programs execute, they exhibit cyclic behavior patterns. Recent research $[2,9,10,27,28,25,11,1,23]$, has shown that it is possible to accurately identify and predict these phases in program execution. Phase behavior can be exploited for accurate architecture simulation [26, 27], to save energy by dynamically reconfiguring caches and processor width $[2,28,10,9]$, to guide compiler optimizations [21,3] and finally to provide feedback to the programmer to guide program optimization. All of these techniques take advantage of the phase behavior that exists in programs.

Prior work on phase classification [27, 17, 28, 25] divides a program's execution into non-overlapping fixed-length intervals. An interval is a contiguous portion of execution (a slice in time) of a program. A phase is a set of intervals within a program's execution that have similar behavior (e.g., IPC, cache miss rates, branch miss rates, etc), regardless of temporal adjacency. This means that intervals that belong to a phase may appear throughout the program's execution. Some of the prior work uses an off-line clustering algorithm to break a program's execution into phases to perform fast and accurate architecture simulation by simulating a single representative portion of each phase of execution [27, 25, 1, 23].

These prior techniques have focused on finding phase behavior in serial applications. In this paper we build upon these prior techniques to find phase behavior in parallel applications. We focus on providing parallel phase analysis for shared memory multi-processors and applications with data parallelism $[22,32,12]$. In this paper, we focus on machines with 2-4 processors because of the expected wide spread deployment of small multi-core machines that will occur over the coming years. There are two main goals with this work. First we propose a parallel program analysis technique that can analyze the phase behavior across different amounts of parallelism (number of threads) for a program/input pair. The primary goal is to enable a user to track how different phases of a program's execution scale across different number of threads to see which phases benefit the most from the additional parallelism. A secondary goal is to exploit the phase characterization for guiding the picking of simulation points for deterministic multi-threaded simulators. This is an enhancement to the single threaded SimPoint [27] algorithm, where we find representative samples across the threads of a parallel program to accurately represent the entire parallel execution on a deterministic multi-threaded simulator like the one used at Intel [29].

The contributions of this paper are:

- We provide a definition of phases of execution for multithreaded workloads. In Section 5 we describe how to recognize similar activities performed by different threads for a program's execution.

- We focus on extracting phase behavior from the execution of a program on native hardware, without using any instrumentation. The sampled information collected with VTune Performance Analyzer is leveraged in order to obtain an estimate of frequencies of instructions executed in each given interval. This allows us to create per-thread code signatures, which are used to phase similar phases across the 
different threads of execution. We perform an analysis to examine the similarity of these parallel phases by studying the standard deviation of performance metrics such as CPI, L2 and L3 cache misses within a phase.

- We propose a parallel program analysis technique that captures the phase behavior for a program/input pair across different number of threads to enable a user to track how different phases of a program's execution scale as the number of threads increases.

- Finally we examine using the parallel phase analysis to pick simulation points to guide deterministic multi-threaded simulation [19, 29].

\section{Related Work}

Program phase behavior can be detected by examining a program's working set [7], and several researchers have examined phase behavior in programs. In this section we give a brief overview of this related work.

Balasubramonian et al [2] proposed using hardware counters to collect miss rates, CPI and branch frequency information for every hundred thousand instructions. They use the miss rate and the total number of branches executed for each interval to dynamically evaluate the program's stability. They used their approach to guide dynamic cache reconfiguration to save energy without sacrificing performance.

Dhodapkar and Smith $[9,10,8]$ found a relationship between phases and instruction working sets, and that phase changes occur when the working set changes. They proposed dynamic reconfiguration of multi-configuration units in response to phase changes indicated by working set changes. They use working set analysis for reconfiguration of instruction cache, data cache and branch predictor to save energy $[9,10]$.

Hind et al. [13] provide a framework for defining and reasoning about program phase classification focusing on how to appropriately define granularity and similarity to perform phase analysis.

In [26, 27], Sherwood et al. proposed that periodic phase behavior in programs can be automatically identified by profiling the code executed. They used techniques from machine learning to classify the execution of the program into phases (clusters). They found that intervals of execution grouped into the same phase had similar behavior across all architectural metrics examined. From this analysis, they created a tool called SimPoint [27], which automatically identifies a small set of intervals of execution (simulation points) in a program for detailed architectural simulation. These simulation points provide an accurate and efficient representation of the complete execution of the program. They then extended this approach to perform hardware phase classification and prediction [28, 18]. In [18], Lau et al. from the same group focus on hardware techniques for accurately classifying and predicting phase changes (transitions). Patil et al. [23] has been examining using SimPoint to pick simulation points to guide simulation at Intel.
Isci and Martonosi $[14,15]$ have shown the ability to dynamically identify the power phase behavior using power vectors. Deusterwald et al. [11] recently used hardware counters and other phase prediction architectures to find phase behavior.

In [6], Davies et. al. used sampled information extracted by the Intel ${ }^{T M}$ VTune Performance Analyzer in order to construct an approximate yet faithful representation of program execution within a given interval. Such information is collected at runtime on native hardware, but is not influenced by the performance of the hardware itself. Performance data is collected at the same time for subsequent validation of results. Analogous methods to SimPoint [27] are then employed to extract Simulation Points. Annavaram et al. [1] used this VTune approach to examine phase behavior for database applications. We use this VTune sampling approach to collect our code vectors, which is detailed in Section 4.

Van Biesbrouck et al. [4] used phase behavior to guide simulation for Simultaneous Multithreading [33]. A co-phase matrix is generated to represent the per-thread performance for each potential combination of the single-threaded phase behaviors that can be found when multiple programs are run together. The co-phase matrix is populated by collecting samples of the programs' phase combinations, and is used to guide fast forwarding between samples. The reason why we do not use the co-phase matrix approach is because it requires too many phase combinations to be evaluated in order to be used in practice. Instead, our approach focuses on identifying the unique phase behaviors seen, and then taking one sample (simulation point) of each phase behavior with whatever else is executing with it at that time to represent that phases execution.

\section{Methodology and Metrics}

The data presented in this paper was collected on a 4-node Itanium II. This platform has four Intel ${ }^{T M}$ Itanium II processors at $1 \mathrm{GHz}$ with a $256 \mathrm{~KB}$ L2 cache and a $3 \mathrm{MB}$ L3 cache, an 870 Intel ${ }^{T M}$ chipset, and a $400 \mathrm{MHz}$ front side bus. This is a multiprocessor system, and each processor has its own L2 and L3 cache, and only main memory is shared across the processors.

Our data collection methodology utilizes the commercially available Intel VTune Performance Analyzer [34]. Our phase analysis framework processes the VTune output file (in tb5 format) collected from the execution of a program on any platform for which VTune is available.

The applications examined in this work are a selection from an extensive set of experiments we ran. We experimented with both OpenMP and p-thread, and on task-parallel applications. We found the definition of phases proved to be coherent even when different threads clearly do not execute the same phase at the same time. To show the range of parallel phase behavior found we provide results for the OpenMP C version of the NAS parallel Benchmarks (NPB) [22], and two more benchmarks OpenMP-parallelized:

- SNP (Single Nucleotide Polymorphism) is capable of detecting structure around a single nucleotide polymorphism in a DNA chain. This application has been coded by 
using Intel's Open Source Probabilistic Network Library (PNL) [32]

- SVM RFE (Support Vector Machine Recursive Feature Elimination [12]) is based on the state of the art Support Vector Machine classification algorithm and is used for eliminating gene redundancy in micro-array data analysis.

Both of these applications repeatedly access very large databases, and apply general purpose machine learning and data mining algorithms to bioinformatics applications.

\subsection{Metrics for Evaluating Phase Classification}

Phase Detection is performed in a completely performanceindependent fashion, solely based on code signatures as described in Section 4. A major assumption underlying this and most of the phase analysis related work is that similar code execution intervals yield similar performance. To this end, we decide to also collect performance data at runtime using VTune while collecting the code signature. This allows us to later verify the assumption and validate our work.

The metrics we examined from VTune are CPI, together with L2 and L3 Hits and Hit-Rates. We have found that these are the key metrics to analyze in order to understand the performance of a multi-threaded application, which is often bounded by issues such as data-reuse and conflicts between caches.

We measure the effectiveness of our phase classifications by examining the similarity of program metrics within each phase. After classifying a program's intervals into phases, we compute the phase based standard deviation for each metric (e.g., CPI, data cache hit rates). This is computed by combining the weighted standard deviation from each phase. We weight each phase's standard deviation by the relative size the phase represents from the entire execution. We compare this phase-based standard deviation to the standard deviation seen when looking at all of the intervals of the program's execution. Better phase classifications will exhibit lower per-phase standard deviation for an architecture metric when compared to the standard deviation of the complete execution. For example, if all of the intervals in the same phase have exactly the same CPI, then the per-phase standard deviation will be zero.

\section{Profiling Program Behavior}

In this section we provide a description of the basic structure we use in order to represent code execution in a given interval.

\subsection{Extended Instruction Pointers}

Davies et al. [6] proposed using hardware sampling of instruction pointers to represent code signatures for finding phase behavior, and such an approach was recently used by Annavaram et al. [1] to try to find phase behavior in database workloads.

In this work, we also use Extended Instruction Pointers (EIPs) to find phase behavior [1]. An EIP is the memory address of an instruction, analogous to PC. The EIPs are extracted while running an application on native hardware. VTune, a commercially available software performance analyzer for Intel ${ }^{T M}$ architectures [34] is used to collect the EIPs. We focus on this approach, instead of instrumentation, since
VTune has the ability to non-intrusively analyze any application running on native hardware with negligible overhead. The underlying VTune driver monitors a large number of performance/code execution attributes stored in the embedded event counters of the Intel processors while a program is being executed on real hardware. It collects information, such as EIPs and CPI, which are then used to perform code clustering, phase analysis, and validation.

VTune interrupts execution at regular intervals of instructions executed and records the EIP and event counter totals (e.g. clock tick count, instruction count.) Sampling at a high frequency can significantly increase execution overhead. Conversely, too low a sampling frequency will lead to sparse data that could compromise phase analysis. Based on our experimental data, we set the VTune sampling rate to be once every hundred thousand instructions. It proved to be a good tradeoff between execution overhead and collecting adequate sampling data. At this sampling rate, the typical overhead of using VTune is not more than $2 \%$.

Annavaram et al. [1] used the EIPs to create an Extended Instruction Pointer Vector (EIPV), which is a one dimensional array where each element in the array corresponds to one Extended Instruction Pointer in the program execution. The EIPV contains all zeroes at the beginning of each interval of execution. During each interval, the number of times each EIP occurs during sampling with VTune is recorded, and each EIP's final interval count is stored in the EIPV.

The intuition behind this is that the behavior of the program at a given time is directly related to the code executed during that interval [27]. The EIP vectors can be used as code signatures for each interval of execution: each vector tells us which portions of code are executed, and how frequently. For a suitably chosen sampling frequency, the sampled information gives a sufficiently accurate estimate of the frequency of execution of significant EIPs within a given interval. By comparing the EIPVs of two intervals, we can evaluate the similarity of those two intervals. If the distance between the EIPVs is small, then the two intervals spend about the same amount of time in roughly the same code, and therefore the performance of those two intervals should be similar.

\subsection{Sampled Basic Block Vectors}

The construction of EIPVs has been proposed in [6] as an alternative to Basic Block Vectors (BBVs), first introduced in [26]. A basic block is a single-entry, single-exit section of code with no internal control flow. Similarly to EIPVs, a $B a$ sic Block Vector (BBV) is a one dimensional array where each element in the array corresponds to one static basic block in the program. During each interval, the number of times each basic block in the program has been entered is counted, and the count is recorded in the BBV.

Lau et al. [16] mapped the EIPs of an EIPV back to coarser program constructs, namely loops and procedures. This improved phase classification when compared to EIPVs for the SPEC CPU2000 suite on IA-32 Linux. This helped reduce the artificial noise between two EIPV code signatures that were classified as different because there were different counts for 
EIPs from the same basic block. Lau et al. [16] proposed mapping the EIPs back to the static code constructs to create Sampled Code Vectors for each interval where each dimension was the number of times each static loop or procedure was sampled.

In this paper, we tried using the sampled loop-proc vectors as in [16], but found that they did not improve performance over EIPVs. Sampled loop-proc vectors worked well for the SPEC 2000 programs, as the programs had enough interesting code behavior spread over the static loops and procedures. However, we found that this was not the case for the parallel benchmarks we examine in this paper, which have only a few static loops and procedures.

Therefore, we examine mapping the EIPs down to basic blocks to create sampled basic block vectors. The mappings in [16] were produced by using Pin to instrument IA-64 binaries. We implemented mapping EIPs to basic blocks using the Itanium version of Pin. We statically process a binary, marking every instruction as a conditional branch, a conditional branch target, both, or neither, and then use these markings as boundaries in assigning a block ID to every instruction in the binary. We then use this ID mapping (a dimension in the sampled BBV) to coalesce all dimensions in the EIPV that map to the same block-ID into a single dimension with weight equal to the summation of the weights of the remapped EIP dimensions, producing a sampled BBV. For the results in this paper we use the sampled BBVs to find the phase behavior in parallel programs.

\section{Discovering Phases for a Single Par- allel Run}

Parallel applications can have multiple threads executing different parts of the binary at the same time. This presents new challenges in program characterization and phase analysis. In this section we provide a detailed description of the algorithm we use that characterizes parallel applications. In this Section we focus on analyzing a single parallel run, and in Section 6 we describe how to extend this to examine behavior varying the number of threads.

\subsection{Phase Analysis Merging All Threads Together}

The foundation of parallel application characterization relies on preserving the parallel structure of execution during phase analysis. The thread level behavior of the application is the framework through which the parallel structure is perceived. Hence, the representation of the thread level behavior is a critical component in the analysis.

One possible thread representation is an agglomerated (combined) view of all the activity across the different threads to create intervals of execution. This is achieved by pooling together the execution samples collected from individual threads into a single execution trace and creating fixed length intervals from that. This trace can be run through existing phase analysis techniques but has the following drawback: the phases discovered in this trace do not apply to any individual threads, but instead apply to the combination of behaviors from all threads.
This provides the following issues: (1) it is difficult to interpret the behavior of individual threads and (2) it is hard to validate the behavior of an agglomerated interval, since it represents a combination of parts of several threads of execution. If threads execute at different rates relative to each other, then the phase representation will not be consistent across the intervals formed. We tried the agglomerated method, and it can provide a coarse parallel characterization, but is not sufficient for low level understanding of the parallel phase behavior.

\subsection{Keeping the Thread Data Separate}

Instead of agglomerating the behavior of the threads, we found that representing each individual thread in the application independently is the key to parallel phase analysis. For this approach each thread has its own set of sampled BBVs for its execution. In this manner each thread is an independent entity. We do this, because we want to find phase similarities across threads.

\subsection{General Algorithm}

The parallel phase analysis algorithm is similar to the serial SimPoint [27] algorithm at a high level. The execution of the application is broken down into intervals which are then clustered into a set of phases. It is different, however, in how it handles the data from multiple threads at different stages in the algorithm. Several modifications are essential to ensure that the phases found between the threads are consistent and can be compared across the threads. The algorithm is described in the following steps:

1. We first collect code execution frequencies for each thread in the application. This data is partitioned into intervals of 100 million instructions, where each thread has a unique set of intervals representing its own execution.

2. For each thread we have a trace of intervals that represents its execution. We then generate a large combined trace for all the threads by concatenating the interval traces from each thread. This step does not contaminate the per thread execution behavior, since each thread occupies a non-overlapping sub-section in the trace. The purpose of this step is to find intervals of execution that are similar across the different threads.

3. We then cluster all the intervals into a set of phases. For this step we use the $k$-means [20] algorithm of SimPoint. SimPoint determines the number of phases by clustering over a range of values, and then use the Bayesian Information Criterion (BIC) [24] to quantify the goodness of each clustering. In this work we considered a range of up to 10 phases, and a larger range can be used for attaining higher accuracy.

This algorithm finds the phases in a parallel execution. Each phase defines a particular code behavior in the parallel execution that is independent of the multi-thread interaction, since we are only looking at the code signatures for the intervals on a per thread basis.

We also find similar execution across threads, since we cluster all of the threads intervals together at the same time. 
The vectors are formed on a per-thread basis, but the clustering is performed looking at all of the intervals from all of the threads at once. Therefore, the phases discovered are applicable across all threads, where similar behavior observed across multiple threads will be captured and characterized as one behavior.

\subsection{Thread Execution Reconstruction}

The phase analysis described above discovers the phase behavior across threads by clustering the BBVs collected in all thread executions in terms of instruction count. We now describe how to map the phase information found to the threaded program's execution over time. In a parallel execution there may be synchronization points, where some threads are waiting for other threads before continuing execution or certain threads are spawned in the middle of execution. We take this into consideration when forming the fixed length interval, so that an interval does not span across these types of stalls. In addition, we need to take this into consideration when mapping the phase classification back to a parallel execution trace.

The goal here is to identify visually for a user what phase of execution each part of a thread's execution is in. VTune outputs samples in the order they were collected across the multiple processors. This provides a complete sequential ordering among the threads of execution. We use this information to reconstruct a total count of instructions retired (global instruction count) across all threads of execution. This allows us to correspond when a fixed length interval, which was assigned to a phase, for a thread occurs during execution relative to the intervals from other threads to examine cross-thread phase behavior over time. Note, since the intervals were formed using only per-thread instruction counts, the start and end of the intervals can be unaligned in terms of the global instruction count with respect to execution time.

Figure 1 and 2 graphically show the phase classification for the NAS benchmarks ft.B and mg.B when using 4 threads with respect to instructions retired over time. The top most sub-figure for each benchmark shows the phases color coded across the entire execution. In these plots, the $x$ axis shows the total number of instructions (global instruction count) retired for all the threads. The global instruction count was not used to perform the phase classification, we just use it to map the per-thread phase intervals to execution time (represented by global instruction count). The $y$-axis is partitioned into 4 sections, 1 per thread. Each phase has a particular color (or shade). If a thread has an interval of white it means that no instructions were retired during that interval. The lower subfigures in each figure are showing L3 cache references, and L3 cache hit rates. The $x$-axis in these sub-figures are showing the number of instructions retired, and are equivalent to the $x$-axis in the top sub-figure for phases.

When mapping a per-thread's intervals to the global instruction count, if there is a large gap (greater than an interval size) in a per-thread's execution with respect to the global instruction count, then a blank (white) interval is shown representing that the thread was stalled or context switched out during that part of execution. It is interesting to see how the phase analysis, performed ignoring any time or similar- ity information among threads, does indeed automatically detect phases coherently with the execution flow across threads. The benchmarks ftB and mgB represented in Figure 1 and 2 are both data-parallel applications, and the phase analysis correctly places all threads in the same phases at the same time for the majority of the execution. However, occasionally different roles for the different threads is also seen; this occurs in the figures whenever one thread is in charge of initialization or collecting results. When this occurred the code signature formed clearly identified that execution as different.

White intervals visible in the picture represent intervals of execution where one or more threads are stalled because a portion of the code is not parallelized or requires a smaller number of threads than the available thread-count. It can also happen for other synchronization issues, or OS activity; note that this naturally happens at the beginning or end of a short serial phase.

\subsection{Single Parallel Run Results}

We now examine the performance of our parallel phase analysis on the NAS benchmark suite and two data mining benchmarks. The programs and methodology used are described in Section 3.

\subsubsection{Reduction of Variance}

The goal of parallel phase analysis is to group together program execution across the different threads by only looking at code signatures. If the phase classification worked well, then the variance in CPI, L2 and L3 cache hit rates should decrease between all of the intervals within a phase when compared to the variance seen across the complete execution of the program. For all of the results an interval size of 100 million instructions and a maximum limit of 10 phases (clusters) was used when performing the phase analysis.

Table 1 shows the mean and standard deviation for CPI (cycles per instruction), and level 2 and level 3 caches hit rates for the full execution. The number in parenthesis is the standard deviation. Results are shown for each program for a 2thread parallel run and a 4-thread run. The last column is the number of phases chosen by our analysis for that run. Note, all of the CPI and hit rate results are the average and standard deviation see across all of the intervals of execution. For example, svm 2-thread has a CPI of 0.87 with a std-dev of 0.32 over all of the intervals of execution, its L3 hit rate is $48 \%$ with a std-dev of $21 \%$, and its execution was clustered into 6 phases.

The NAS benchmark suite shows several different potential program behaviors, where ep. B and is. B are at the two extremes. In Table 1, the program is. B has a huge variance in CPI (+/- 10) for 4-thread because there are intervals of execution that have a spike greater than $10 \mathrm{CPI}$. The serial part of the code for is. B covers approximately $80 \%$ of the execution (instructions retired), and this makes it a very peculiar one among the NAS benchmarks. In comparison, the results for ep . B show that across all of execution there is a low standard deviation across all of the metrics.

Table 1 shows that the CPI can be stable or increase when going from 2-threads to 4-threads. This happens whenever the 

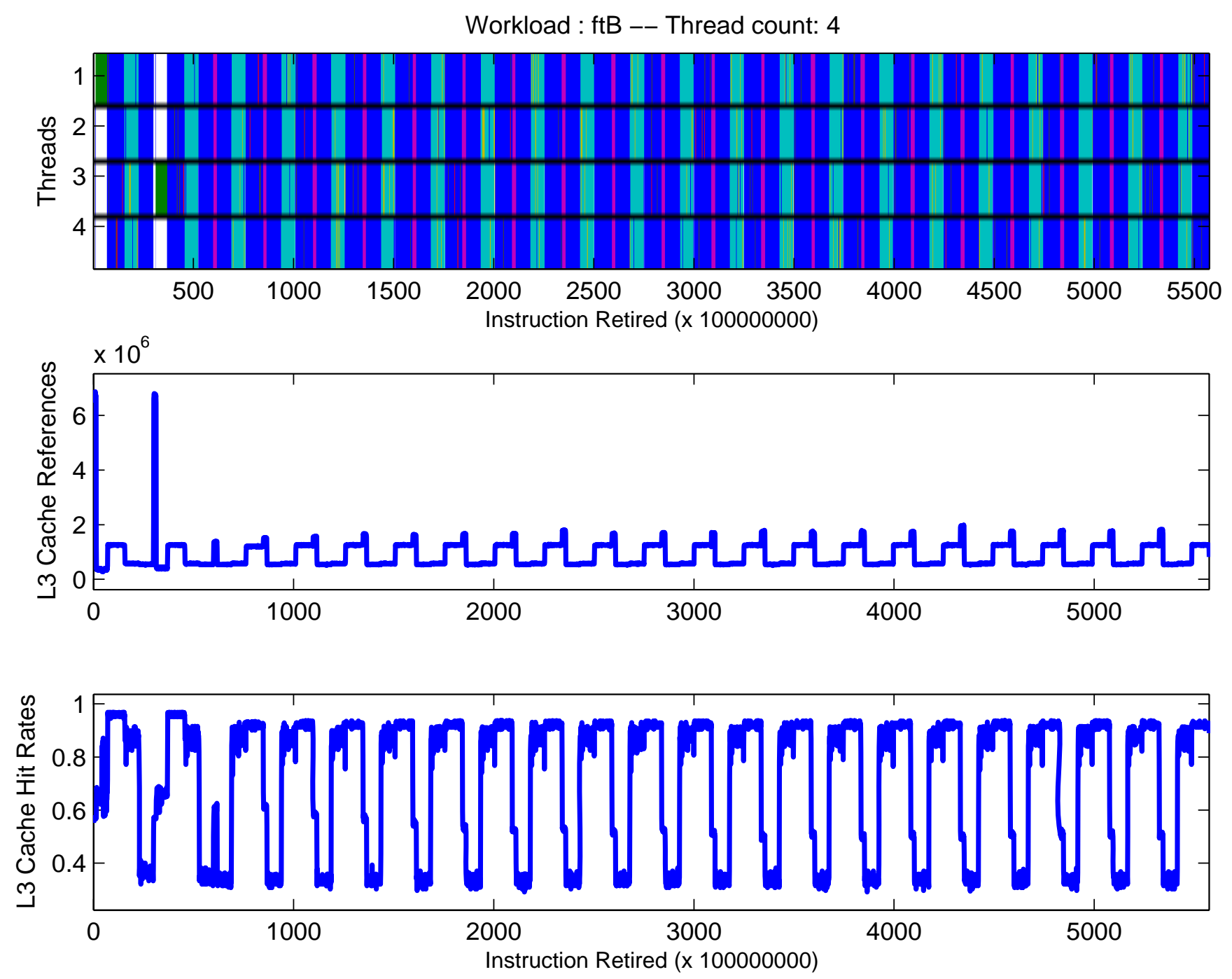

Figure 1: Phase classifications and L3 performance metrics for a four-threaded run of ftB. Phase classifications are applied to each thread independently.

speedup obtained by executing the benchmark in parallel does not scale linearly with the thread count, and the parallelization introduces overhead. The CPI shown is calculated on a perthread basis, and does not represent a measure of speed of execution of the overall program on the machine, but rather a measure of speed of execution of instructions on each single thread.

We now examine how well the phase classification, based on code, worked in terms of the underlying architecture metrics. If the phase analysis groups the intervals correctly, then we should see reductions in the standard deviation of these architecture metrics, when examining them across all of the intervals within a phase. Figures 3,4 and 5 show the reduction in the standard deviation of CPI, L2 and L3 hit rates over the baseline values shown in Table 1 for the 2-thread and 4thread runs. This is computed by first computing the difference between the standard deviation of the baseline and the weighted standard deviation of the phases, and then dividing it by the baseline standard deviation. A large reduction in standard deviation means that the phase analysis succeeds in breaking varying program behavior into homogeneous phases. The results show that when looking at the program's execution in terms of phases that on average the std-dev for CPI is reduced by $50 \%$, the $\mathrm{L} 2$ hit rate by $60 \%$ and the $\mathrm{L} 3$ hit rate by $45 \%$. The reason why there is little reduction in the std-dev for ep. B for L2 and L3 hit rates is that there was little std-dev to begin with as shown in Table 1 .

\section{Discovering Phases Across Parallel Runs}

One of the motivations for us to perform phase analysis for parallel programs it to be able to examine the same behavior and performance when using a different number of threads. This can be used by programmers and scientists to study where they should tune their code and to better understand the implications of increasing the number of processors to run an application on. For example, to analyze the benefit of parallelizing a program we would like to take a representative slice of the program's execution when using 2 threads, and that same 

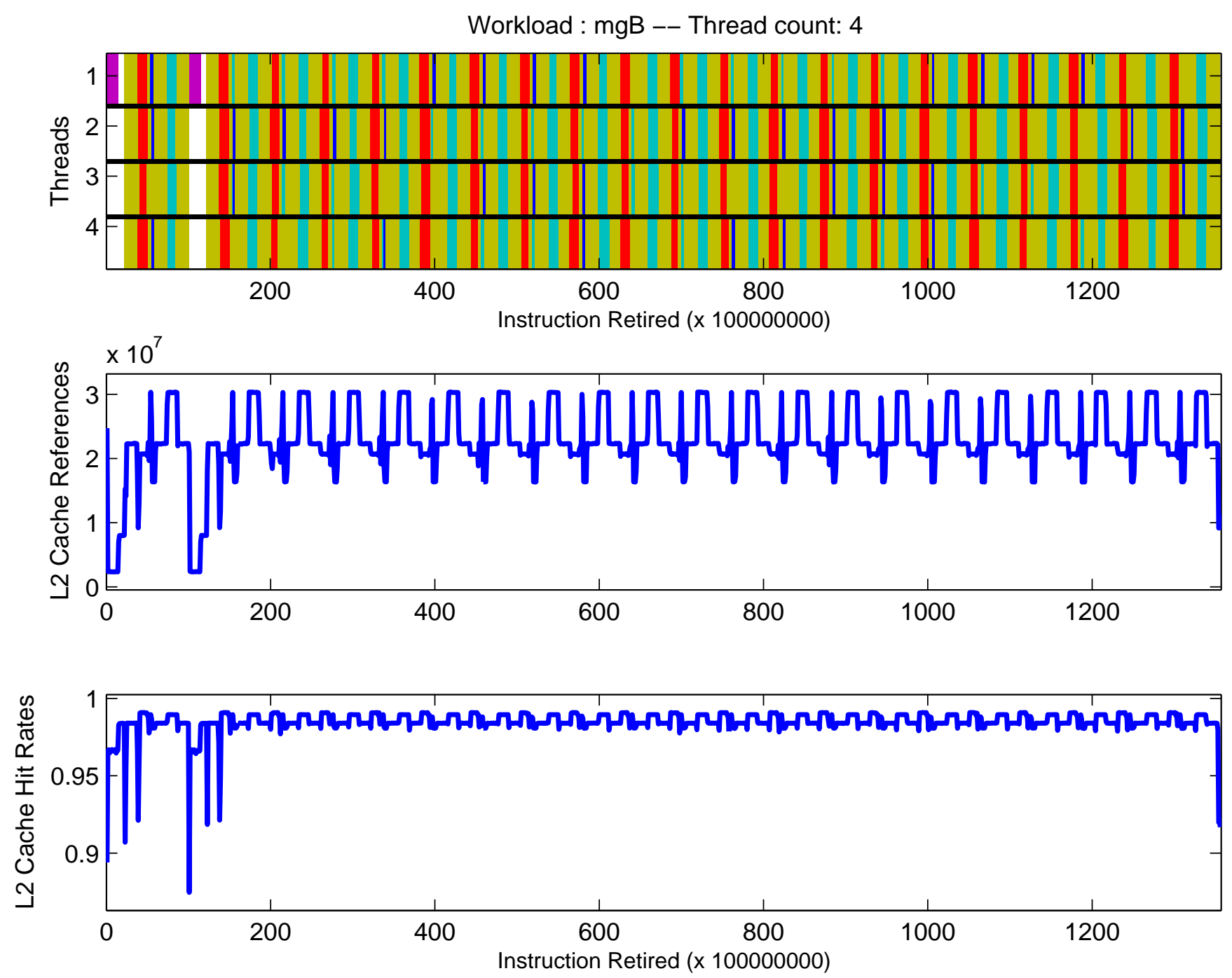

Figure 2: Phase classifications and L2 performance metrics for a four-threaded run of $m g B$. Phase classifications are applied to each thread independently.

exact slice for 3 threads, 4 threads, etc... and compare how the programs' CPI or cache hit rates change as we vary the number of threads. Prior work on the scalability of parallel applications $[30,5,31]$ has focused on how the overall execution of the program scales as the number of threads varies. In comparison, we are instead focusing on the scalability of the program in terms of how each of its phases scales as the number of threads varies.

In this section, we describe how we achieve this "threadvarying" phase analysis by extending the parallel phase analysis described in Section 5.

\subsection{Phase Analysis Varying the Number of Threads}

The prior section described how to find phases in a single parallel run of a program. The goal of this analysis is to find similar intervals of execution between different runs as the number of threads is varied. We call this thread-varying phase analysis.

We want to perform this analysis on a parallel program running a specific input varying the number of threads. We start by combining all of the sampled BBVs for each thread for a given run as described in Section 5. We then concatenate each of these run's (where only the number of threads has been varied) sampled BBVs together for a specific parallel binary/input. SimPoint phase analysis is then run over this vector trace. This results in a clustering that successfully groups together not only intervals from separate threads of the same run (as shown in the prior section), but also intervals from different runs (where the threads were varied).

\subsection{Thread-Varying Phase Analysis Results}

In this work we applied our thread-varying phase analysis on four separate runs for each program: serial, 2 threads, 3 threads, and 4 threads. We combined the runs as described, and computed the phases across the threads and runs. In doing this thread-varying phase analysis, we verified that the intervals grouped within the same phase from the same run had similar architecture metrics as found in the previous section. But intervals from different runs (different number of threads) grouped into the same phase will not have the same architecture metrics. This is exactly what we want to analyze. We use this thread-varying analysis to see for similar code regions 


\begin{tabular}{|l||r|r|r|r|r|}
\hline Benchmark & \# Threads & CPI & L2 Hit Rate & L3 Hit Rate & \# Phases \\
\hline \hline bt.A & 2 & $0.93(+/-0.40)$ & $0.98(+/-0.02)$ & $0.51(+/-0.10)$ & 6 \\
& 4 & $1.08(+/-0.44)$ & $0.98(+/-0.01)$ & $0.53(+/-0.10)$ & 5 \\
\hline cg.B & 2 & $1.36(+/-0.60)$ & $0.65(+/-0.02)$ & $0.87(+/-0.03)$ & 7 \\
& 4 & $1.45(+/-1.05)$ & $0.66(+/-0.02)$ & $0.87(+/-0.02)$ & 5 \\
\hline ep.B & 2 & $0.99(+/-0.01)$ & $0.99(+/-0.00)$ & $0.98(+/-0.04)$ & 5 \\
& 4 & $0.99(+/-0.01)$ & $0.99(+/-0.00)$ & $1.00(+/-0.01)$ & 5 \\
\hline ft.B & 2 & $0.73(+/-1.58)$ & $0.95(+/-0.04)$ & $0.87(+/-0.14)$ & 6 \\
& 4 & $0.82(+/-2.60)$ & $0.97(+/-0.05)$ & $0.75(+/-0.25)$ & 9 \\
\hline is.B & 2 & $3.04(+/-5.77)$ & $0.83(+/-0.13)$ & $0.75(+/-0.33)$ & 8 \\
& 4 & $4.15(+/-10.04)$ & $0.82(+/-0.12)$ & $0.74(+/-0.33)$ & 4 \\
\hline lu.B & 2 & $1.07(+/-0.24)$ & $0.95(+/-0.02)$ & $0.49(+/-0.10)$ & 4 \\
& 4 & $0.98(+/-0.20)$ & $0.95(+/-0.01)$ & $0.69(+/-0.11)$ & 4 \\
\hline mg.B & 2 & $0.81(+/-1.27)$ & $0.99(+/-0.01)$ & $0.49(+/-0.08)$ & 6 \\
& 4 & $0.94(+/-1.90)$ & $0.99(+/-0.01)$ & $0.46(+/-0.08)$ & 8 \\
\hline sp.A & 2 & $1.61(+/-0.25)$ & $0.96(+/-0.00)$ & $0.47(+/-0.05)$ & 9 \\
& 4 & $2.19(+/-0.34)$ & $0.96(+/-0.00)$ & $0.46(+/-0.02)$ & 7 \\
\hline snp & 2 & $0.96(+/-0.09)$ & $0.95(+/-0.02)$ & $0.06(+/-0.07)$ & 8 \\
& 4 & $0.93(+/-0.05)$ & $0.94(+/-0.03)$ & $0.36(+/-0.21)$ & 6 \\
\hline svm & 2 & $0.87(+/-0.32)$ & $0.91(+/-0.05)$ & $0.48(+/-0.21)$ & 6 \\
& 4 & $1.40(+/-0.41)$ & $0.90(+/-0.04)$ & $0.48(+/-0.18)$ & 5 \\
\hline \hline Avg & & $1.36(+/-1.38)$ & $0.92(+/-0.03)$ & $0.61(+/-0.12)$ & 6 \\
\hline
\end{tabular}

Table 1: Full execution CPI, L2 and L3 hit rates with standard deviation across all intervals of execution. The number of phases is equivalent to the number of simulation points.

how the architecture metrics varied for a phase as the number of threads was varied.

To show this, we will examine the execution of snp running with a single thread (serial execution), as well as 2,3 , and 4 threads. Then we combined the threads in each of the parallel runs and perform our thread-varying phase analysis. Note that the number of phases chosen is different from Section 5 since we are performing the thread-varying clustering.

Figure 6 shows the phases discovered across four different parallel executions for snp. The top most sub-figure displays the phases in a single threaded run of snp. The next two sub-figures below show phases in a two threaded run. The next three sub-figures are for a three threaded run, and the last four sub-figures are a four threaded run. The $\mathrm{x}$-axis shows the phase classification results over the global count of all instructions executed across all threads. Each phase is denoted by a different color or gray-scale (as shown on the right side of the Figure), and the same phase colors are used across the different runs. White means that no instructions are executed during that interval due to synchronization or serialization.

Figure 6 shows that even though each run has a different number of threads, we are able to identify the same regions of execution across the different runs. The initialization phase for snp is the shade of the first phase in the single threaded execution. Exactly one thread has that color and all other threads are inactive during that part of execution for the multi-threaded runs. It is also worth noting that the phases also line up along the $x$-axis. In this figure the $x$-axis is the number of instructions retired across all threads in a run, and this means that the phases found between different runs execute a similar number of instructions.
Figure 7 shows the number of cycles per phase across 4 different parallel executions of sp. A. The Y-axis is number of cycles and $\mathrm{X}$-axis is the different runs varying the number of threads 1, 2, 3 and 4. In this Figure, the number of cycles (Y-axis) is the actual time spent executing the benchmark. Cycles are not accounted for on a per-thread basis, as it was in the CPI computation of Section 5, but actually represent the time elapsed while one or more threads is in a specific phase. If two or more threads are in two different phases during a part of execution, the time elapsed is split among them with appropriate weights. For example, Phase 2 (the top line) accounts for 11 billion cycles of execution with one thread, and only 3.8 billion cycles when four threads are used.

This Figure is an example of the coherency of our phase definition. Increasing thread-count improves the performance of each phase for this application. An intuitive downward trend is visible for each phase, however the trend varies from phase to phase. One can see that phase 2 and phase 4 (the top 2 lines) benefit the most from the parallelization, and one can go back to the code to analyze why this is the case. It also shows that more significant speedups can be achieved by parallelizing Phase 2 and 4 up to four threads, whereas Phase 5 has diminishing returns from parallelization once two threads are used. This is a confirmation that it is worthwhile to perform phase analysis on parallel benchmarks, as each phase exhibit different parallelization potential and performance.

Figure 8 shows the number of instructions retired that are classified into each of the phases across 4 different parallel runs (again, 1, 2, 3, and 4 threaded executions). This shows that across the different runs, each phase occupies a similar number of executed instructions. For example, for Phase 2 

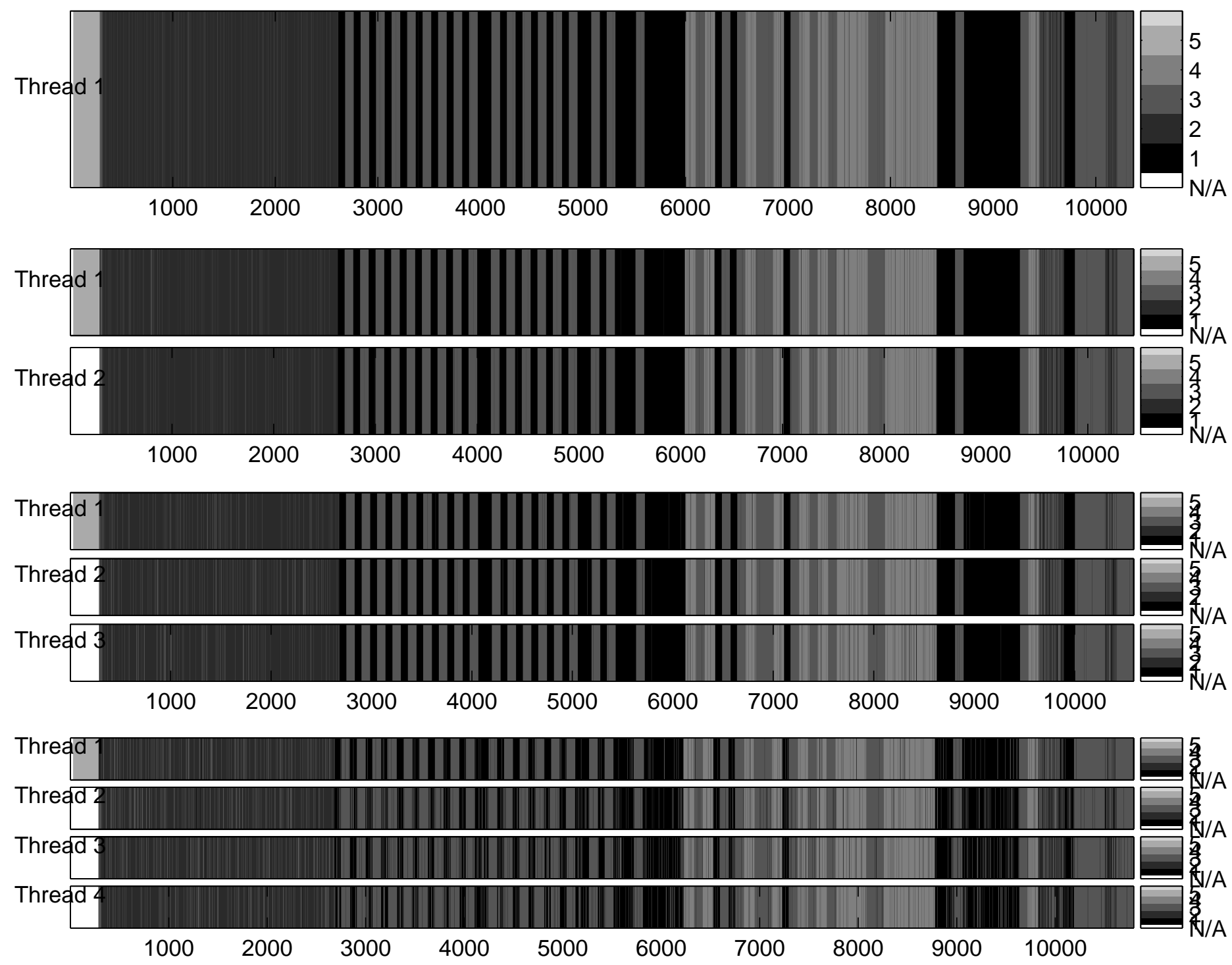

Figure 6: Phases discovered across 4 different parallel executions of snp: serial, 2, 3, and 4 threads. The x-axis shows the phase classification results over the global count of all instructions executed across all threads.

(the top line), the total number of instructions executed is 80 billion with one thread, and about 85 billion for 4 threads.

The important observation here is that the proportion of intervals assigned to each phase is the same across the different runs when varying the threads. This shows that phase behaviors coherently correspond to the execution of different paths in the code. The thread-count increase changes the distribution of the execution of these paths among different threads, but does not significantly alter their nature nor the amount of time spent in each phase. It is therefore to be expected and required for a good definition of phases that a given phase behavior occupies approximately the same number of instruction retired, independently from the thread-count, as the number of threads is varied.

\section{Parallel SimPoint}

We now examine using the phase groupings described in Section 5 for Parallel SimPoint. The goal is to choose a small set of simulation points (on a per thread basis) that when simulated on a deterministic multi-thread simulator [19, 29] provide an accurate representation of the complete parallel run.

A simulation infrastructure used at Intel [29] deterministically models the Intel Pentium 4 with the capability to simulate threaded workloads in both single processor and multiprocessor configurations. It employs the UserLit tool [29] for collecting traces from an executable. A trace consists of a checkpoint of the user code address space at a specific point in execution, as well as system call logs from that point onward. A UserLit trace allows deterministic simulation of a multi-threaded program starting at the checkpoint, consuming the system call logs as they occur during execution. Note, only deterministic multi-threaded execution is supported. If some non-deterministic behavior occurs, which is detected by difference in the system call log and simulated execution, then the simulation is aborted, since only simulating programs with deterministic re-execution (using a system call log) is supported. To gather these UserLit traces, engineers have picked by hand 


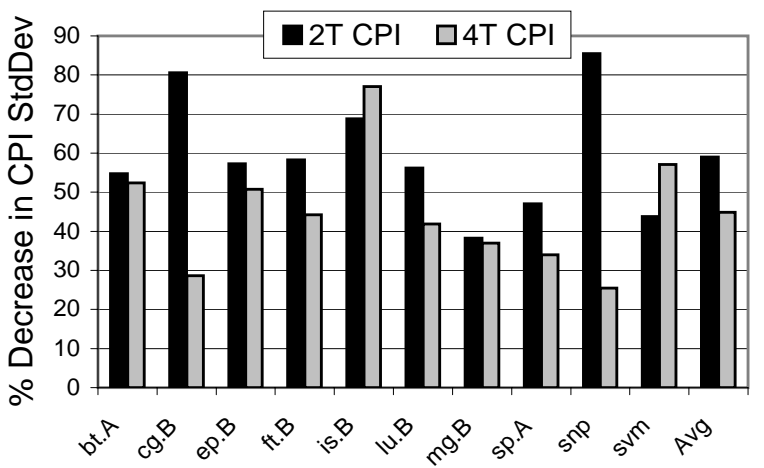

Figure 3: Percent reduction in standard deviation for CPI with phase analysis

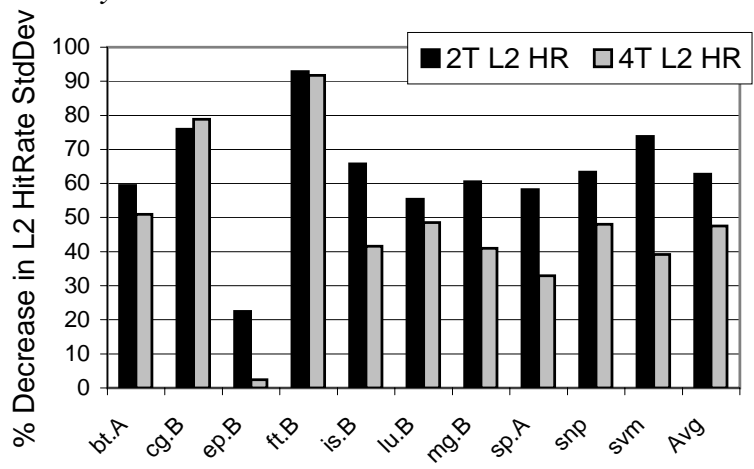

Figure 4: Percent reduction in standard deviation for L2 hit rate with phase analysis

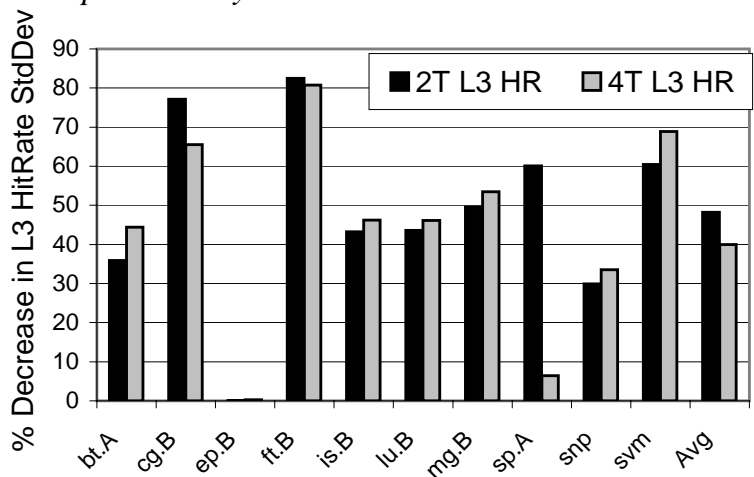

Figure 5: Percent reduction in standard deviation for L3 hit rate with phase analysis

which parts of the program's execution to trace. Our goal is to instead use SimPoint [27] to guide where the UserLit traces should be taken.

For Parallel SimPoint, we use the phase clustering algorithm described in Section 5. A simulation point is chosen for each phase, which is the interval for a specific thread with its sampled basic block vector closest to the centroid of the phase. Each simulation point is assigned a weight equal to the percent of the program's execution (in terms of intervals) its phase represents. The architecture metrics from these simulation point intervals can then be combined to accurately represent the program's overall metric. See [27] for a complete description of SimPoint.

Once simulation points are chosen, a UserLit checkpoint and trace would be gathered to represent the parallel execution going on during that intervals execution. This includes

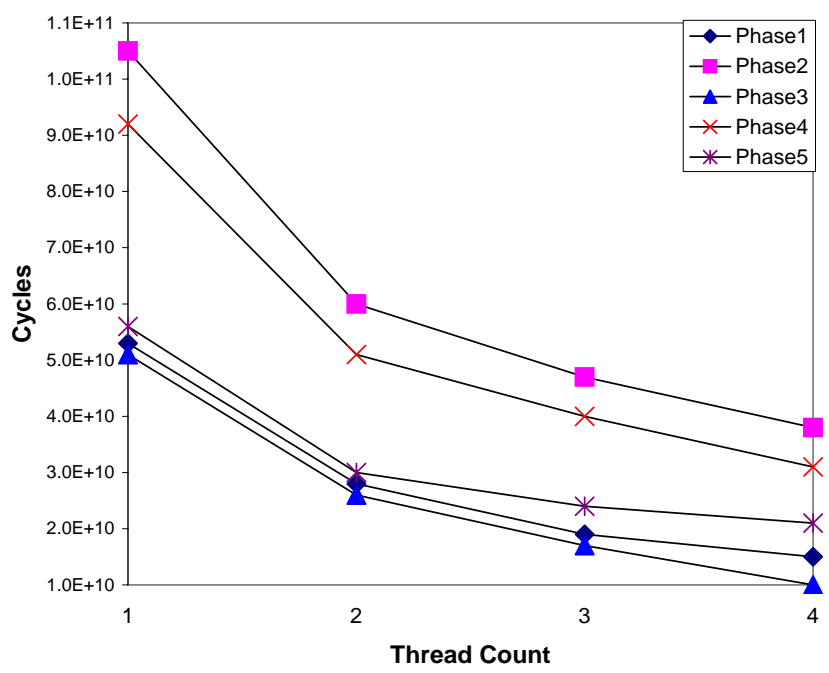

Figure 7: Number of cycles per phase across 4 different parallel executions of sp.A: serial, 2, 3, and 4 threads.

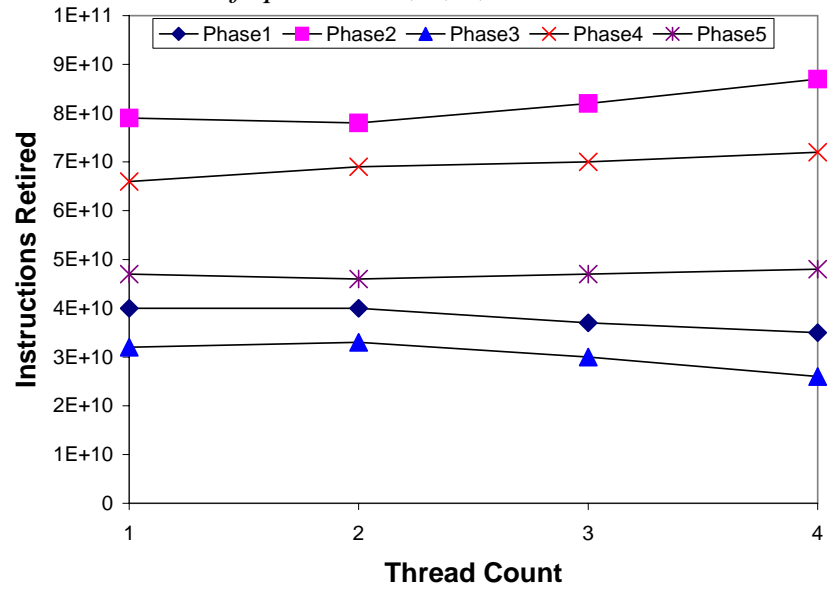

Figure 8: Number of instructions per phase across 4 different parallel executions of sp.A: serial, 2, 3, and 4 threads.

the activities of all of the threads running during that simulation point's interval of execution. Then performance statistics are collected via detailed simulation for each simulation point UserLit checkpoint/log. The performance statistics for each simulation point are then weighted by the number of instructions that are executed within the phase belonging to that simulation point. The entire execution performance statistics are estimated by combining the weighted simulation point statistics. This allows the UserLit traces to be chosen automatically, instead of choosing them by hand, which is the current approach.

Figure 9 shows the relative error rates for CPI and L2 hit rate for the 2-thread and 4-thread runs when comparing the parallel SimPoint estimated metric to the overall program's baseline metric. The number of simulation points used for each program is shown in Table 1 . The results show that the CPI error is $15 \%$ or less, with an average of $3 \%$ for the 2thread runs, and similar results are seen for the cache hit rate. Lower error rates are seen for the 4-thread runs. Programs like is. B have higher error rates due to the huge deviation in program behavior between the parallel and sequential part of execution. Even so, the error rate is small (less than 4\%) for 
4-threads. This result, along with the reduction in std-dev as shown in Section 5, shows that our approach groups similar parts of execution together based only on the sampled code signatures.

It is important to note that the absolute error of a program/input run on one hardware configuration is not as important as tracking the change in metrics across different architecture configurations. When using simulation points for an architecture design space exploration the CPI error compared to the baseline is not as important as making sure that this error is consistent between the different architectures being examined. What is important is that a consistent relative error is seen across the design space exploration, and SimPoint has this consistent bias as shown in [25]. Therefore, even though a program/input using SimPoint might have 10\% error when compared to the complete execution, we have found that when aggressively varying the design space that we see this same relative error in the same direction. This allows the designer, when using SimPoint, to make the correct design space exploration trade-offs. This is because the relative error is consistent and biased in the same direction.

\section{Summary}

In this paper we focus on discovering phases in parallel applications running on shared memory systems. We start by describing how to recognize similar activities performed by different threads for a program's execution. The results showed that this can be used with SimPoint to accurately represent the programs parallel behavior with an average error less than $4 \%$ for CPI, and L2 and L3 hit rates, as well as significantly reduce the std-dev of these metrics within a phase.

We also showed that we could perform thread-varying phase analysis across different runs of a program as the number of threads used varies from 1 to 4 threads. We found that thread-varying phase analysis can be used to examine the effect on specific parts of the program's execution as the number of threads is varied. This can be used by researchers to better understand a parallel program's execution for different number of threads/processors. Finally we showed that using the parallel phase analysis can be used to accurately pick simulation points to guide deterministic multi-threaded simulation [29].

\section{Acknowledgments}

This work was funded in part by NSF grant No. CCR0311710, NSF grant No. ACR-0342522, UC MICRO grant No. 03-010, and a grant from Intel.

\section{References}

[1] M. Annavaram, R. Rakvic, M. Polito, R. Hankins, J.Y. Bouguet, and B. Davies. The fuzzy correlation between code and performance predictability. In 37th International Symposium on Microarchitecture, December 2004.

[2] R. Balasubramonian, D. H. Albonesi, A. Buyuktosunoglu, and S. Dwarkadas. Memory hierarchy reconfiguration for energy and performance in general-purpose processor architectures. In 33rd International Symposium on Microarchitecture, pages 245-257, 2000.
[3] R. D. Barnes, E. M. Nystrom, M. C. Merten, and W. W. Hwu. Vacuum packing: Extracting hardware-detected program phases for post-link optimization. In 35th International Symposium on Microarchitecture, December 2002.

[4] M. V. Biesbrouck, T. Sherwood, and B. Calder. A co-phase matrix to guide simultaneous multithreading simulation. In IEEE International Symposium on Performance Analysis of Systems and Software, March 2004.

[5] Dhruba Chandra, Fei Guo, Seongbeom Kim, and Yan Solihin. Predicting inter-thread cache contention on a chip multi-processor architecture. In HPCA '05: Proceedings of the 11th International Symposium on HighPerformance Computer Architecture, pages 340-351, Washington, DC, USA, 2005. IEEE Computer Society.

[6] B. Davies, J.Y. Bouguet, M. Polito, and M. Annavaram. ipart: An automated phase detection and recognition tool. Technical report, Intel Research Tech Report IRTR-2004-1 (http://research.intel.com/ir/tools/presentations/files/IR-TR2004-1-iPART.pdf), 2003.

[7] P.J. Denning and S. C. Schwartz. Properties of the working-set model. Communications of the ACM, 15(3):191-198, March 1972.

[8] A. Dhodapkar and J. Smith. Comparing program phase detection techniques. In 36th International Symposium on Microarchitecture, December 2003 .

[9] A. Dhodapkar and J. E. Smith. Dynamic microarchitecture adaptation via co-designed virtual machines. In International Solid State Circuits Conference, February 2002.

[10] A. Dhodapkar and J. E. Smith. Managing multi-configuration hardware via dynamic working set analysis. In 29th Annual International Symposium on Computer Architecture, May 2002.

[11] E. Duesterwald, C. Cascaval, and S. Dwarkadas. Characterizing and predicting program behavior and its variability. In International Conference on Parallel Architectures and Compilation Techniques, October 2003.

[12] Isabelle Guyon, Jason Weston, Stephen Barnhill, and Vladimir Vapnik. Gene selection for cancer classification using support vector machines. Machine Learning, 36(1-2):389-422, April 2002.

[13] M. Hind, V. Rjan, and P. Sweeney. Phase shift detection: A problem classification. Technical report, IBM, August 2003.

[14] C. Isci and M. Martonosi. Identifying program power phase behavior using power vectors. In Workshop on Workload Characterization, September 2003 .

[15] C. Isci and M. Martonosi. Runtime power monitoring in high-end processors: Methodology and empirical data. In 36th International Symposium on Microarchitecture, December 2003.

[16] J. Lau, J. Sampson, E. Perelman, G. Hamerly, and B. Calder. The strong correlation between code signatures and performance. In Proceedings of the 2005 IEEE International Symposium on Performance Analysis of Systems and Software, March 2005.

[17] J. Lau., S. Schoenmackers, and B. Calder. Structures for phase classification. In IEEE International Symposium on Performance Analysis of Systems and Software, March 2004.

[18] J. Lau, S. Schoenmackers, and B. Calder. Transition phase classification and prediction. In Proceedings of the International Symposium on HighPerformance Computer Architecture, January 2005.

[19] K. Lepak, H. Cain, and M. Lipasti. Redeeming ipc as a performance metric for multithreaded programs. In 12th International Conference on Parallel Architectures and Compilation Techniques, 2003.

[20] J. MacQueen. Some methods for classification and analysis of multivariate observations. In L. M. LeCam and J. Neyman, editors, Proceedings of the Fifth Berkeley Symposium on Mathematical Statistics and Probability, volume 1, pages 281-297, Berkeley, CA, 1967. University of California Press.

[21] M. Merten, A. Trick, R. Barnes, E. Nystrom, C. George, J. Gyllenhaal, and Wen mei W. Hwu. An architectural framework for run-time optimization. IEEE Transactions on Computers, 50(6):567-589, June 2001.

[22] http://phase.hpcc.jp/omni/benchmarks/npb/.

[23] H. Patil, R. Cohn, M. Charney, R. Kapoor, A. Sun, and A. Karunanidhi. Pinpointing representative portions of large intel itanium programs with dynamic instrumentation. In MICRO, 2004. 


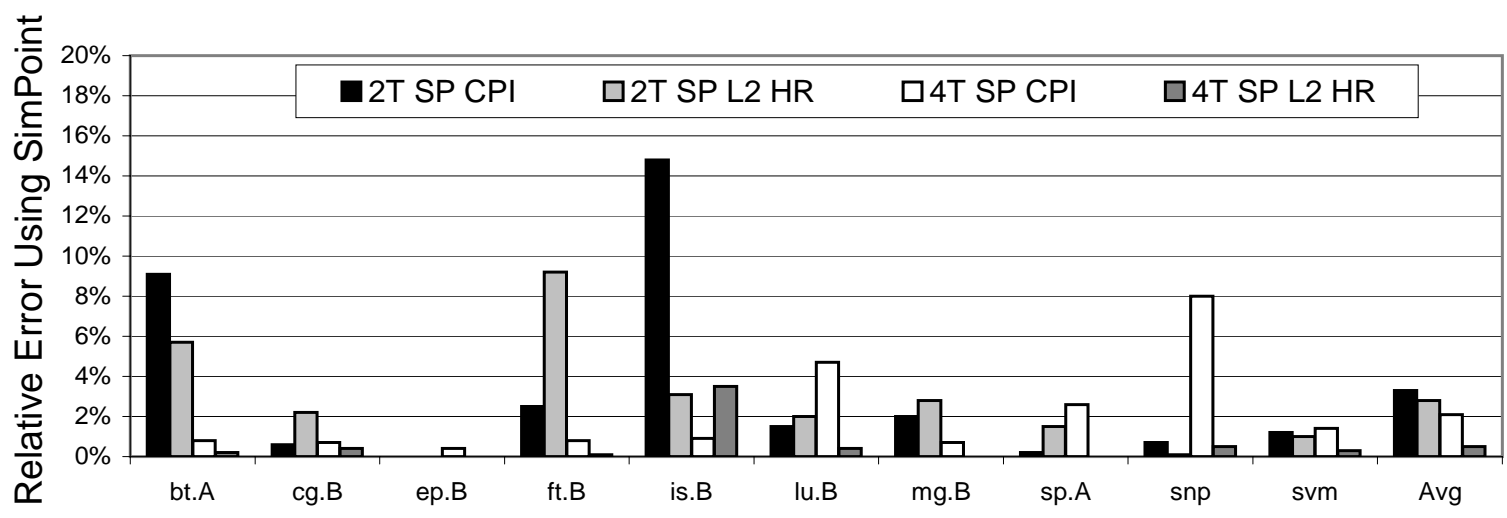

Figure 9: SimPoint relative error rates for CPI, L2 and L3 hit rates

[24] D. Pelleg and A. Moore. $X$-means: Extending $K$-means with efficient estimation of the number of clusters. In Proceedings of the 17th International Conf. on Machine Learning, pages 727-734. Morgan Kaufmann, San Francisco, CA, 2000.

[25] E. Perelman, G. Hamerly, and B. Calder. Picking statistically valid and early simulation points. In International Conference on Parallel Architectures and Compilation Techniques, September 2003.

[26] T. Sherwood, E. Perelman, and B. Calder. Basic block distribution analysis to find periodic behavior and simulation points in applications. In International Conference on Parallel Architectures and Compilation Techniques, September 2001.

[27] T. Sherwood, E. Perelman, G. Hamerly, and B. Calder. Automatically characterizing large scale program behavior. In Proceedings of the 10th International Conference on Architectural Support for Programming Languages and Operating Systems, October 2002.

[28] T. Sherwood, S. Sair, and B. Calder. Phase tracking and prediction. In 30th Annual International Symposium on Computer Architecture, June 2003.

[29] R. Singhal, K.S. Venkatraman, E. Cohn, J.G. Holm, D.Koufaty, M.J. Lin, M. Madhav, M. Mattwandel, N. Nidhi, J. Pearce, and M. Seshadri. Performance analysis and validation of the intel pentium 4 processor on $90 \mathrm{~nm}$ technology. Intel Technology Journal (ftp://download.intel.com/technology/itj/2004/volume08issue01/art03 performance/vol8iss L art03.pdf), 8(1), February 2004.

[30] Anand Sivasubramaniam, Aman Singla, Umakishore Ramachandran, and $\mathrm{H}$. Venkateswaran. An approach to scalability study of shared memory parallel systems. SIGMETRICS Perform. Eval. Rev., 22(1):171-180, 1994.

[31] Allan Snavely, Laura Carrington, Nicole Wolter, Jesus Labarta, Rosa Badia, and Avi Purkayastha. A framework for performance modeling and prediction. In Supercomputing '02: Proceedings of the 2002 ACM/IEEE conference on Supercomputing, pages 1-17, Los Alamitos, CA, USA, 2002. IEEE Computer Society Press.

[32] http://www.intel.com/research/mrl/pnl/.

[33] Dean Tullsen, Susan Eggers, and Henry Levy. Simultaneous multithreading: Maximizing on-chip parallelism. In Proceedings of the 22rd Annual International Symposium on Computer Architecture (ISCA), June 1995.

[34] http://www.intel.com/software/products/vtune/. 